\title{
Toroidal equilibria in spherical coordinates
}

\author{
K.H. Tsui \\ Instituto de Física - Universidade Federal Fluminense, \\ Campus da Praia Vermelha, Av. General Milton Tavares de Souza s/n \\ Gragoatá, 24.210-346, Niterói, Rio de Janeiro, Brasil. \\ tsui@if.uff.br
}

Received __; accepted _ 


\begin{abstract}
The standard Grad-Shafranov equation for axisymmetric toroidal plasma equilibrium is customary expressed in cylindrical coordinates with toroidal contours, and through which benchmark equilibria are solved. An alternative approach to cast the Grad-Shafranov equation in spherical coordinates is presented. This equation, in spherical coordinates, is examined for toroidal solutions to describe low $\beta$ Solovev and high $\beta$ plasma equilibria in terms of elementary functions.
\end{abstract}

Subject headings: Toroidal Equilibria 


\section{Grad-Shafranov Equation}

The Grad-Shafranov equation $(1,2)$ for toroidal plasma equilibria is traditionally formulated in a toroidal system using cylindrical coordinates to suit the plasma topology. This equation describes the poloidal magnetic flux, in cylindrical coordinates, with source terms that are themselves flux gradients. By specifying the source functionals in magnetic flux, toroidal equilibria are solved by different methods. Because of the cylindrical representation of a toroidal topology, the solutions are not easy to visualize and the mathematical analytic tools are rather limilted, which weaken the communication between theorists and experimentalists. Here, we propose to solve axisymmetric plasma equilibria in standard spherical coordiantes by looking for solutions with toroidal topology. With the familiar spherical system, and the large volume of well-known special functions in this coordinate system, the solutions in toroidal topology appear in a much friendly presentation, which improves physical interpretations.

We first derive the Grad-Shafranov equation in spherical coordinates by considering

$$
\begin{gathered}
\vec{J} \times \vec{B}-\nabla p=0, \\
\nabla \times \vec{B}=\mu \vec{J} .
\end{gathered}
$$

With axisymmetry, the magnetic field and the current density can be represented by two scalar functions in standard spherical coordinates

$$
\begin{array}{r}
\vec{B}=\nabla P \times \nabla \phi+Q \nabla \phi=\frac{1}{r \sin \theta}\left\{+\frac{1}{r} \frac{\partial P}{\partial \theta},-\frac{\partial P}{\partial r},+Q\right\} \\
\mu \vec{J}=\frac{1}{r \sin \theta}\left\{+\frac{1}{r} \frac{\partial Q}{\partial \theta},-\frac{\partial Q}{\partial r},-\frac{\partial^{2} P}{\partial r^{2}}-\frac{1}{r^{2}} \sin \theta \frac{\partial}{\partial \theta}\left(\frac{1}{\sin \theta} \frac{\partial P}{\partial \theta}\right)\right\} .
\end{array}
$$


Making use of Eq. 2 to eliminate the current density, Eq. 1 renders three components. The $\phi$ component contains only the magnetic force, and it is

$$
\begin{gathered}
\frac{\partial P}{\partial r} \frac{\partial Q}{\partial \theta}-\frac{\partial P}{\partial \theta} \frac{\partial Q}{\partial r}=0 \\
Q(r, \theta)=Q(P(r, \theta)) .
\end{gathered}
$$

As for the $\theta$ component, it reads

$$
\frac{\partial P}{\partial \theta}\left\{\frac{\partial^{2} P}{\partial r^{2}}+\frac{1}{r^{2}} \sin \theta \frac{\partial}{\partial \theta}\left(\frac{1}{\sin \theta} \frac{\partial P}{\partial \theta}\right)\right\}+Q \frac{\partial Q}{\partial \theta}+\mu r^{2} \sin ^{2} \theta \frac{\partial p}{\partial \theta}=0
$$

Since the spatial dependencies of $Q(r, \theta)$ is through a functional of $P(r, \theta)$, we could express the $\theta$ dependency in plasma pressure by $P$ to write $p(r, \theta)=p(r, P)$, and the above equation becomes

$$
\begin{array}{r}
\left\{\frac{\partial^{2} P}{\partial r^{2}}+\frac{1}{r^{2}} \sin \theta \frac{\partial}{\partial \theta}\left(\frac{1}{\sin \theta} \frac{\partial P}{\partial \theta}\right)+\frac{\partial Q}{\partial P} Q\right\}+\mu r^{2} \sin ^{2} \theta \frac{\partial p}{\partial P} \\
=\left\{\frac{\partial^{2} P}{\partial r^{2}}+\frac{1}{r^{2}} \sin \theta \frac{\partial}{\partial \theta}\left(\frac{1}{\sin \theta} \frac{\partial P}{\partial \theta}\right)+\frac{1}{2} \frac{\partial Q^{2}}{\partial P}\right\}+\mu r^{2} \sin ^{2} \theta \frac{\partial p}{\partial P}=0 .
\end{array}
$$

This equation is the Grad-Shafranov equation $(1,2)$ of axisymmetric toroidal plasma equilibrium, represented in spherical coordinate system. The first three terms represent the nonlinear force-free field with $\mu \vec{J}=K(P) \vec{B}$, where $K(P)=\partial Q / \partial P$ is a scalar function. This can be verified from Eq. 3 and Eq. 4 when we impose $\mu \vec{J}=K(P) \vec{B}$. In particular, we would have the linear force-free field should we take $Q(P)=a P$ with constant $K(P)=a$. The last term is the plasma pressure balance. The magnetic function $Q^{2}(P)$ and the 
pressure function $p(P)$ are source functions of the Grad-Shafranov equation that need to be specified. Finally, the $r$ component of Eq. 1 reads

$$
\begin{array}{r}
\frac{d}{d r} p(r, P)=-\frac{1}{\mu}\left(\frac{1}{r \sin \theta}\right)^{2} \frac{\partial P}{\partial r}\left\{\frac{\partial^{2} P}{\partial r^{2}}+\frac{1}{r^{2}} \sin \theta \frac{\partial}{\partial \theta}\left(\frac{1}{\sin \theta} \frac{\partial P}{\partial \theta}\right)+\frac{1}{2} \frac{\partial Q^{2}}{\partial P}\right\} \\
=\frac{\partial P}{\partial r} \frac{\partial}{\partial P} p(r, P)
\end{array}
$$

The term $d p(r, P) / d r$ on the left side refers to the total radial derivative, which has an explicit and an implicit part, and the right side of this radial equation is the magnetic force. Making use of the $\theta$ component, Eq. 7, the right side is equal to the implicit part of the radial pressure gradient, which cancels the same term on the left side, the implicit part of the radial derivative. The radial component, therefore, reads $\partial p / \partial r=0$, and the plasma pressure is a function of $P$ only,

$$
p=p(P) \text {. }
$$




\section{Low $\beta$ Equilibria}

To solve Eq. 7 analytically, we take the source functions as

$$
\begin{gathered}
Q^{2}(P)=2 b^{2} P+a^{2} P^{2}+Q_{0}^{2}, \\
p(P)=p_{0} P+p_{0}^{\prime} \ln P-C_{0},
\end{gathered}
$$

where $p_{0}$ and $p_{0}^{\prime}$ carry the dimension of pressure, $a^{2}$ and $b^{2}$ measure the dimension of magnetic field, $Q_{0}^{2}$ and $C_{0}$ are constants, and $P(r, \theta)$ is a dimensionless function. With $a^{2}=0$ and $p_{0}^{\prime}=0$, Solovev (3) solved the Grad-Shafranov equation for low $\beta$ equilibria with linear source functions. These source functions have been extended by other investigators $(4,5)$. With $a^{2} \neq 0$, the quadratic form for $Q^{2}$ plus a likewise quadratic form for $p$, without the $\ln P$ term, have been analyzed by many authors in different ways (6-9). In particular, $\mathrm{Hu}$ has considered a cubic form for $Q^{2}(10)$. On the other hand, numerical routines have also been advanced by many authors $(11,12)$. Here, we include a $\ln P$ term in the plasma pressure function, and regard Eq. 10a and Eq. 10b as the generalized Solovev case, and solve for the Grad-Shafranov equation in spherical coordinates for a low $\beta$ plasma. The $\theta$ component, therefore, becomes

$$
\left\{r^{2} \frac{\partial^{2} P}{\partial r^{2}}+\sin \theta \frac{\partial}{\partial \theta}\left(\frac{1}{\sin \theta} \frac{\partial P}{\partial \theta}\right)+(a r)^{2} P+(b r)^{2}\right\}+\mu p_{0} r^{4} \sin ^{2} \theta+\mu p_{0}^{\prime} r^{4} \sin ^{2} \theta \frac{1}{P}=0
$$

Dividing this equation over by $P$, separating the variables by writing $P(r, \theta)=R(r) \Theta(\theta)$, 
denoting $x=\cos \theta$, and with $n(n+1)$ as the separation constant, we have

$$
\begin{gathered}
\left(1-x^{2}\right) \frac{d^{2} \Theta(x)}{d x^{2}}+n(n+1) \Theta(x)=-\frac{(b r)^{2}}{R(r)}-\frac{\mu p_{0}^{\prime}}{b^{4}} \frac{(b r)^{4}}{R^{2}(r)} \frac{\left(1-x^{2}\right)}{\Theta(x)}, \\
r^{2} \frac{d^{2} R(r)}{d r^{2}}-n(n+1) R(r)=-(a r)^{2} R(r)-\frac{\mu p_{0}}{b^{4}}(b r)^{4} \frac{\left(1-x^{2}\right)}{\Theta(x)} .
\end{gathered}
$$

These equations can be solved by iterations, because of the low $\beta$ plasma. We, therefore, first solve the homogeneous equations with the right sides null giving

$$
\begin{gathered}
\Theta_{0}(x)=\left(1-x^{2}\right) \frac{d P_{n}(x)}{d x}, \\
R_{0}(r)=A_{0}(b r)^{n+1},
\end{gathered}
$$

where $P_{n}(x)$ is the Legendre polynomial. The coefficient $A_{0}$ reflects the amplitude of the magnetic flux through Eq. 3. To iterate on the zeroth order solutions, we take $n=1$ and substitute Eq. 13a and Eq. 13b to the right sides of Eq. 12a and Eq. 12b to get

$$
\begin{array}{r}
\left(1-x^{2}\right) \frac{d^{2} \Theta_{1}(x)}{d x^{2}}+n(n+1) \Theta_{1}(x)= \\
=-\frac{(b r)^{2}}{R_{0}(r)}-\frac{\mu p_{0}^{\prime}}{b^{4}} \frac{(b r)^{4}}{R_{0}^{2}(r)} \frac{\left(1-x^{2}\right)}{\Theta_{0}(x)} \\
\left.r^{2} \frac{d^{2} R_{1}(r)}{d r^{2}}-n(n+1) R_{1}(r)=-(a r)^{2} R_{0}(r)-\frac{\mu p_{0}}{A_{0}^{2}}\right\}=-\frac{1}{b_{0}^{*}}(b r)^{4} \frac{\left(1-x^{2}\right)}{\Theta_{0}(x)} \\
=-\left\{A_{0}\left(\frac{a}{b}\right)^{2}+\frac{\mu p_{0}}{b^{4}}\right\}(b r)^{4}=-A_{1}(b r)^{4} .
\end{array}
$$


By simple inspection, the first order solutions and the general solutions are

$$
\begin{array}{r}
\Theta_{1}(x)=-\frac{1}{2 A_{0}^{*}}, \\
\Theta(x)=\left(1-x^{2}\right)-\frac{1}{2 A_{0}^{*}}, \\
R_{1}(r)=-\frac{A_{1}}{10}(b r)^{4}, \\
R(r)=A_{0}(b r)^{2}-\frac{A_{1}}{10}(b r)^{4} .
\end{array}
$$

We see that $\Theta(x)$ has a lobed solution centered at $x=0$ with a maximum amplitude $\left(1-1 / 2 A_{0}^{*}\right)$. The radial solution $R(r)$ is composed of two terms. The first term is positive and increases to the second power of $(b r)$, while the second term is negative and increases to the fourth power of $(b r)$. The radial function $R(r)$ is positive at small $(b r)$ and negative at large $(b r)$. It vanishes at $(b r)^{2}=0$ and $(b r)^{2}=10 A_{0} / A_{1}$, with a maximum in between. This maximum of $R(r)$ and the lobed solution of $\Theta(x)$ are essential for a torus structure for the magnetic flux. With the given source functions, we notice that the iteration scheme can not be carried beyond the first round. Consequently, if Eq. 15a and Eq. 15b have any meaning at all, $R_{1}(r)$ has to be much less than $R_{0}(r)$, and $\Theta_{1}(x)$ has to be much less than $\Theta_{0}(x)$, leading to $A_{1} / A_{0}<<1$ and $1 / A_{0}^{*}<<1$. This requires $\left[(a / b)^{2}+\left(\mu p_{0} / b^{4} A_{0}\right)\right]<<1$, or $(a / b)^{2}<<1$ and $\left(\mu p_{0} / b^{4} A_{0}\right)<<1$, and $\left[1+\left(\mu p_{0}^{\prime} / b^{4} A_{0}\right)\right]<<A_{0}$, for a low $\beta$ plasma. 


\section{Magnetic Torus}

With the spatial structure solved, the magnetic field components are given by

$$
\begin{array}{r}
B_{r}=+\frac{1}{r \sin \theta} \frac{1}{r} \frac{\partial P}{\partial \theta}=-\frac{1}{r^{2}} R(r) \frac{d \Theta(x)}{d x}, \\
B_{\theta}=-\frac{1}{r \sin \theta} \frac{\partial P}{\partial r}=-\frac{1}{r} \frac{d R(r)}{d r} \frac{1}{\left(1-x^{2}\right)^{1 / 2}} \Theta(x), \\
B_{\phi}=+\frac{1}{r \sin \theta} Q(P) .
\end{array}
$$

The solution $R(r)$ vanishes at some $r$ where we have $B_{r}(r)=0$. The solution $\Theta(x)$ also vanishes at some $x$. Together they describe the magnetic fields. Within this region of $(r, x)$, the topological center defined by $d R(r) / d r=0$ and $d \Theta(x) / d x=0$ has $B_{r}=0$ and $B_{\theta}=0$. This is the magnetic axis, $r=r_{*}$, where the magnetic field is entirely toroidal. The field lines about this center are given by

$$
\frac{B_{r}}{d r}=\frac{B_{\theta}}{r d \theta}=\frac{B_{\phi}}{r \sin \theta d \phi} .
$$

By axisymmetry, the third group is decoupled from the first two groups. For the field lines on an $(r-\theta)$ plane, we consider the first equality between $B_{r}$ and $B_{\theta}$ which gives $P=R(r) \Theta(x)$ equals to a constant or

$$
P(r, x)=R(r) \Theta(x)=C .
$$

In other words, the nested poloidal field lines are given by the contours of $P(r, x)$ on the $(r-x)$ plane. At the topological center, we have $\Theta(x)$ maximum and $R(r)$ maximum, so 
that $P(r, x)$ is maximum. Since $r \sin \theta$ is the distance of a point on the $(r-x)$ plane to the $\mathrm{z}$ axis, Eq. 16c states that the line integral of $B_{\phi}$ around the circle on the azimuthal plane is measured by $2 \pi Q$,

$$
2 \pi r \sin \theta B_{\phi}=2 \pi Q=\mu I_{z}
$$

and the center has the maximum of this line integral about the axis of symmetry. Also, it is evident that $Q$ is equivalent to the axial current, where the constant part $Q=Q_{0}$ amounts to a uniform current. As for $P$, we evaluate the poloidal magnetic flux by integrating Eq. 16b on the $x=0$ plane over a cross section to give

$$
\int_{r_{*}}^{r} 2 \pi r B_{\theta} d r=-2 \pi\left(P(z)-P\left(z_{*}\right)\right)
$$




\section{Near Nonlinear Force-Free High $\beta$ Equilibria}

For high $\beta$ plasmas, we take source functions

$$
\begin{gathered}
Q^{2}(P)=a^{2} P^{2}+Q_{0}^{2}, \\
p(P)=p_{0} P-C_{0} .
\end{gathered}
$$

The Grad-Shafranov equation, Eq. 7, reads

$$
\left\{r^{2} \frac{\partial^{2} P}{\partial r^{2}}+\sin \theta \frac{\partial}{\partial \theta}\left(\frac{1}{\sin \theta} \frac{\partial P}{\partial \theta}\right)+(a r)^{2} P\right\}+\mu p_{0} r^{4} \sin ^{2} \theta=0
$$

The first three terms correspond to the force-free magnetic field as can be verified by setting $\mu \vec{J}=a \vec{B}$ between Eq. 3 and Eq. 4, and the last term is the plasma pressure balance term. Separating the variables gives

$$
\begin{array}{r}
\left(1-x^{2}\right) \frac{d^{2} \Theta(x)}{d x^{2}}+n(n+1) \Theta(x)=0 \\
r^{2} \frac{d^{2} R}{d r^{2}}+\left[(a r)^{2}-n(n+1)\right] R=-\left(\frac{\mu p_{0}}{a^{4}}\right)(a r)^{4} \frac{\left(1-x^{2}\right)}{\Theta}
\end{array}
$$

The first equation gives

$$
\Theta(x)=\left(1-x^{2}\right) \frac{d P_{n}(x)}{d x}=\left(1-x^{2}\right)
$$


where $n=1$ is again chosen. This solution gives $\Theta(x)$ positive for any $x$, which is appropriate for small aspect ratio plasmas. As for the second equation, having $n=1$, the solution is given by $R(r)=R_{0}(r)+R_{1}(r)$, where $R_{0}(r)$ and $R_{1}(r)$ are the homogeneous and particular solutions. The homogeneous solution is described by

$$
R_{0}(r)=A_{0} \operatorname{arj}_{n}(a r)=A_{0} z j_{n}(z)
$$

where $j_{n}(z)$ is the spherical Bessel function. As for the particular solution, denoting $A_{1}=\left(\mu p_{0} / a^{4}\right)$, we have

$$
\begin{array}{r}
z^{2} \frac{d^{2} R_{1}}{d z^{2}}+\left[z^{2}-n(n+1)\right] R_{1}=-A_{1} z^{4}, \\
R_{1}(r)=-A_{1}(a r)^{2}=-A_{1} z^{2} .
\end{array}
$$

We note that the homogeneous solutions, $R_{0}(r)$ and $\Theta(x)$, correspond to the linear or nonlinear force-free solutions of the first three terms of Eq. 20 with null or finite $Q_{0}^{2}$ respectively. The last term for plasma pressure appears only in the particular solution, $R_{1}(r)$, that keeps the pressure balance. The homogeneous radial solution is an oscillating function in $z=a r$, which has sucessive maxima, and the homogeneous meridian solution has a lobe peaked at $x=0$, like the low $\beta$ case. The superposition of the particular radial solution only slightly modifies the homogeneous solutions. We could use the region between $z=0$ and the first root of $j_{n}(z)$, with $n=1$, to describe low aspect ratio high $\beta$ toroidal plasma equilibria. Since Eq. 22, Eq. 23, and Eq. 24 are exact solutions of Eq. 20, with source functions of Eq. 19a and Eq. 19b, these exact solutions decribe high $\beta$ plasmas, that are nearly force-free. It is interesting to note that exact near force-free solutions appear in high $\beta$ equilibria, while the low $\beta$ equilibria are not force-free. 


\section{Low Aspect Ratio Fusion Tori}

These benchmark analytic equilibria are most relevant for current international collaboration scale fusion tokamaks, such as JET and ITER, with D shaped plasma cross section. The homogeneous radial force-free solution $R_{0}(r)$ is shown in Fig.1 with $A_{0}=0.05 \mathrm{Tm}^{2}$ taken arbitrarily. This $R_{0}(r)$ solution has the first null point at $z_{0}=4.5$. To consider the particular solution $R_{1}(r)$, we first have to determine the constant $A_{1}=\left(\mu p_{0} / a^{4}\right)$. To fix the parameter $a$, we remark that the first stationary point, for the magnetic axis, is around

$$
z_{*}=a r_{*}=2.7 \text {. }
$$

Taking the major radius $r_{*}=0.5 \mathrm{~m}$ for a given low aspect ratio spherical tokamak, we would have $a=z_{*} / r_{*}=5.4 / \mathrm{m}$. Considering plasma pressure of $10^{5} \mathrm{~Pa}$, we have $A_{1}=1.3 \times 10^{-9} p_{0}=1.3 \times 10^{-4}$. With the given $A_{1}$, the particular solution of Eq. 24 is

shown in Fig.2. Superimposing the two solutions gives the near force-free general solution $R(z)=R_{0}(z)+R_{1}(z)$ which shifts the null point of $R(z)$ to $z_{0}<4.5$, where the plasma pressure vanishes. The corresponding contour point of $z_{0}<4.5$ on the inner side of the torus is $z_{0}=0$, where the plasma pressure also vanishes. However, there are engineering aspects of fusion torus, such as divertor scrape-off effect, that alter the outer and inner $z_{0}$ plasma boundaries. This effectively means that the plasma boundary is set by the constant $C_{0}$ in Eq. 19b or Eq. 10b determined by engineering considerations.

The poloidal magnetic contours of Eq. 18 are shown in Fig.3. Since the plasma pressure of Eq. 19b is expressed in terms of $P$, it shares the same D shaped contours of Fig.3. As for the toroidal field, with $Q_{0}^{2}=0$, the toroidal field circulation about the $\mathrm{z}$ axis is expressed in 
terms of $2 \pi Q=2 \pi a P$, and therefore it also shares the contours of Fig.3. As for the current density of Eq. 4, making use of the Grad-Shafranov equation of Eq. 20 gives

$$
\begin{array}{r}
\mu \vec{J}=\frac{1}{r \sin \theta}\left\{+\frac{1}{r} \frac{\partial Q}{\partial \theta},-\frac{\partial Q}{\partial r},+\left(a^{2} R_{0}+a^{2} R_{1}+\mu p_{0} r^{2}\right) \Theta\right\} \\
=\frac{1}{r \sin \theta}\left\{+\frac{1}{r} \frac{\partial Q}{\partial \theta},-\frac{\partial Q}{\partial r},+a^{2} R_{0} \Theta\right\}
\end{array}
$$

The middle equality is obtained by cancelling the $R_{1}$ term with the $\mu p_{0}$ term using Eq. 24. Analogous to the magnetic field lines, the current density field lines are given by

$$
\frac{J_{r}}{d r}=\frac{J_{\theta}}{r d \theta}=\frac{J_{\phi}}{r \sin \theta d \phi}
$$

Considering the first equality, the poloidal current density contours are given by

$$
Q(r, x)=C
$$

As a result of this, Fig.3 also represents the poloidal current density contours in the linear force-free case of $Q_{0}^{2}=0$ such that $Q(P)=a P(r, x)=a R(r) \Theta(x)$.

The $B_{\phi}$ field along $x=\cos \theta=0$ in Tesla, Eq. 16c, is shown in Fig.4. Since this field scales as $R(r) / r$, it has a $j_{1}(r)$ profile of Fig.1, and it peaks around $z=2.1$. The $B_{\theta}$ field along $x=\cos \theta=0$ in Tesla, Eq. 16b, is also shown in Fig.4. This component scales as $(1 / r)(d R(r) / d r)$, and it crosses zero around $z=z_{*}=2.6$ which sets the magnetic axis. We note that the magnetic axis at $z=2.6$, where $B_{\theta}$ vanishes, and the stationary point of the toroidal field at $z=2.1$, where $B_{\phi}$ is maximum, do not coincide. They are separated by $\Delta z=a \Delta r=0.5$, or $\Delta r=0.1 \mathrm{~m}$. Since the toroidal field contours are off-centered 
from the poloidal field contours, the magnetic pressure contours are not aligned with the plasma pressure contours. The discrepancy is due to the magnetic curvature which gives rise to magnetic tension according to $\mu \vec{J} \times \vec{B}=(\vec{B} \cdot \nabla) \vec{B}-\nabla B^{2} / 2$. This difference $\Delta z$ would be reduced if we use subsequent second and third peaks of $R_{0}(r)=6.1,9.3$ and corresponding $j_{1}(r)=6.0,9.2$, not shown in Fig.1. As an example, for the second peak, we would have $z_{*}=6.1$ and $\Delta z=0.1$. With major radius $r_{*}=2 \mathrm{~m}$, this would give $\Delta r=\left(\Delta z / z_{*}\right) r_{*}=0.03 \mathrm{~m}$, and would have high aspect ratio equilibria. The toroidal and poloidal current densities, $J_{\phi}$ and $J_{\theta}$, along $x=\cos \theta=0$ in $10^{6} \mathrm{~A} / \mathrm{m}^{2}$ are shown in Fig.5. Besides the scale difference, Fig.4 and Fig.5 are slightly different with $J_{\phi} / B_{\phi}$ slightly larger than $J_{\theta} / B_{\theta}$. Had not been the plasma pressure dependent particular solution $R_{1}(z)$, they would be the same, because of the force-free nature with $\vec{J}$ and $\vec{B}$ being proportional. 


\section{Safety Factor}

One important parameter that measures the quality of equilibrium is the safety factor, which measures the number of turns that the toroidal field circles about the toroidal axis of symmetry through the major radius as the poloidal field makes one turn about the magnetic axis through the minor radius returning to the same poloidal angle, but not necessarily the same toroidal angle. In spherical coordinates, this amounts to the winding number of the toroidal field about the $\mathrm{z}$ axis of symmetry as the poloidal field circles one complete turn about the magnetic axis. With our spherical coordinates, the poloidal field is composed of $B_{r}$ and $B_{\theta}$, and the usual safety factor expression does not apply. In order to rederive an expression for the safety factor, we consider the field line equation, Eq. 17. Since $B_{r}$ and $B_{\theta}$ are constrained by the poloidal field line contours, we need only one of them to track $B_{\phi}$ around the torus. By considering the radial component, and writing $Q_{0}^{2}=a^{2} \gamma^{2}$, such that $Q(P)=a\left(P^{2}+\gamma^{2}\right)^{1 / 2}$, the field line around the torus is given by

$$
\begin{aligned}
2 \pi q\left(z_{1}\right)=2 \pi q\left(z_{2}\right)= & d \phi=\oint \frac{B_{\phi}}{B_{r}} \frac{d r}{r \sin \theta}=\oint \frac{Q(P)}{\partial P / \partial \theta} \frac{d r}{r \sin \theta} \\
& =\frac{1}{2} \oint \frac{\left[R^{2}(z)\left(1-x^{2}(z)\right)^{2}+\gamma^{2}\right]^{1 / 2}}{R(z)\left(1-x^{2}(z)\right)} \frac{d z}{x(z)} \\
& =\oint_{z_{1}}^{z_{2}} \frac{\left[R^{2}(z)\left(1-x^{2}(z)\right)^{2}+\gamma^{2}\right]^{1 / 2}}{R(z)\left(1-x^{2}(z)\right)} \frac{d z}{x(z)}
\end{aligned}
$$

where the integration is carried out over the contour $R(z) \Theta(x)=C$, as in Fig.3, with $x(z)$ in the integrant given by

$$
x(z)^{2}=\left(1-\frac{C}{R(z)}\right)=\frac{1}{R(z)}(R(z)-C) .
$$

The integration is bounded by $z_{1}<z<z_{2}$ on the $x=0$ horizontal plane, where 
$x_{1}=x\left(z_{1}\right)=0$ and $x_{2}=x\left(z_{2}\right)=0$ are the first and second intercepts of a given contour on the horizontal axis. Although the integrant is singular at the bounds $z_{1}$ and $z_{2}$, it is integrable. As the contour value $C$ increases towards the maximum of $R(z)$, the contour closes onto the magnetic axis of $z=z_{*}$, and $x_{1}$ and $x_{2}$ come closer together towards $z_{*}$. In this limit, $x(z)$ in the denominator approaches zero, and the integration interval $z_{1}$ and $z_{2}$ of Eq. 28 also reduces to null. As a result, the contour integral near the magnetic axis depends on the limiting integral of the last factor $d z / x(z)$. To get the correct limit, we Taylor expand $R(z)$ about $z_{*}$ to second order to obtain

$$
2 \pi q\left(z_{*}\right)=\frac{\left[R^{2}\left(z_{*}\right)+\gamma^{2}\right]^{1 / 2}}{R\left(z_{*}\right)} \oint_{z_{1}}^{z_{2}} \frac{d z}{x(z)}=\left\{\frac{2\left[R^{2}\left(z_{*}\right)+\gamma^{2}\right]}{R\left(z_{*}\right)\left|\partial^{2} R\left(z_{*}\right) / \partial z^{2}\right|}\right\}^{1 / 2} \ln \frac{\left|z_{2}-z_{*}\right|}{\left|z_{1}-z_{*}\right|}
$$

Since the $R(z)$ profile is not symmetric around $z_{*}$, as shown in Fig.1, $z_{1}$ and $z_{2}$ of a given contour value do not approach $z_{*}$ at equal intervals, and $2 \pi q\left(z_{*}\right)$ is finite. Since the second order term of the Taylor expansion is a symmetric quadratic term in $\left(z-z_{*}\right)$, this left-right non-symmetry of the maximum comes from the third order cubic term.

As an example, let us consider $\gamma^{2}=0.01$. Since the maximum value of $P=R \Theta$ is about 0.05 , according to Fig. 1 with $\Theta=1$, the constant $\gamma^{2}$ term dominates the $P^{2}$ term in $Q$. By Eq. 19a, this amounts to an almost uniform axial current $\mu I_{z}=2 \pi Q$. In this case, the scalar $K(P)=\partial Q / \partial P$ is not a constant, but a function of $P$, which makes the force-free equation nonlinear. The difference between the linear and nonlinear force-free fields is that the toroidal magnetic field $B_{\phi}$ vanishes at some radial positions for linear case, while it is always poisitive definite for nonlinear case. With the corresponding parameters in Figs.1-5, the safety factor profile is shown in Fig.6, which ressembles well with laboratory profiles, having a basin within the plasma interior surrounded by high values on the border. The value at the border can be lowered by reducing the constant $\gamma^{2}$. 


\section{Conclusions}

We have examined the axisymmetric toroidal plasma equilibria for low and high $\beta$ plasmas. The center of the spherical system, $z=0$, has been excluded because this point usually lies outside the plasma domain due to the machine vessel. However, for spheromaks, the center is connected to the plasma domain. In this case, our solutions provide hollow profiles for plasma pressure. Rather than solving the Grad-Shafranov equation in toroidal geometry with cylindrical coordinates, devised to envelope axisymmetric toroidal plasmas, we have taken a different approach to represent the Grad-Shafranov equation in spherical coordinates and have solved for toroidal solutions. This approach allows benchmark plasma equilibria, such as the generalized Solovev type low $\beta$ equilibrium and the standard high $\beta$ equilibrium, be solved by separation of variables in terms of elementary special functions, and provides easier visualization of the solutions. The essential features of the solutions in spherical coordinates are that the radial function $R(z)$ has a maximum at $z=z_{*} \neq 0$, and the meridian function $\Theta(x)$ has a lobe at $x=0$. These two features assure the function $P(z, x)=R(z) \Theta(x)$ an axisymmetric structure of a torus. Realistic high $\beta$ near force-free D shaped contours are evident in these analytic representations.

In the generalized Solovev type source functions, the equilibrium is solved approximately by sucessive approximation to the first order. As a result, the validity of the solution requires strictly a low $\beta$ plasma. For more specific source functions, the equilibrium is solved exactly in closed form. The solution is, therefore, valid for high $\beta$ plasmas. Surprisingly, the high $\beta$ exact solution is constructed on a force-free magnetic field superimposed by a high $\beta$ plasma contribution. Although with high $\beta$, the plasma contribution is small comparing to the force-free part, making the overall solution near force-free. The reason for this near force-free solution to confine plasmas is that it relies basically on the tensional force of 
the magnetic field line curvature, not the scalar magnetic pressure, to balance the plasma pressure. This curvature is evident because the magnetic axis where $B_{\theta}=0$ is significantly displaced from the maximum of $B_{\phi}$. For this reason, the magnetic field profile remains near force-free, while plasmas are confined. Such strong curvature is inherent from low aspect ratio plasmas. The absence of such curvature in high aspect ratio plasmas obliges the machines to operate in low $\beta$ limit with magnetic fields away from force-free configuration. 


\section{REFERENCES}

1 -H. Grad and H. Rubin, Proceedings of the Second United Nations Conference on the Peaceful Uses of Atomic Energy, Vol. 21, P. 190, (United Nations, Geneva, 1958).

2 -V.D. Shafranov, Sov. Phys. JETP 6, 545 (1958).

3 -L.S. Solovev, Sov. Phys. JETP 26, 400 (1968).

4 -S.B. Zheng, A.J. Wootton, and E.R. Solano, Phys. Plasmas 3, 1176-1178 (1996).

5 -B. Shi, Phys. Plasmas 12, 122504 (2005).

6 -E. Mazzucato, Phys. Plasmas 3, 441-443 (1996).

7 -P.J. Mc Carthy, Phys. Plasmas 6, 3554-3560 (1999).

8 -C.V. Atanasiu, S. Gunter, K. Lackner, and I.G. Miron, Phys. Plasmas 11, 3510-3518 (2004).

9 -L. Guazzotto and J.P. Freidberg, Phys. Plasmas 14, 112508 (2007).

10 -Y. Hu, Phys. Plasmas 15, 022505 (2008).

11 -L.L. LoDestro and L.D. Pearlstein, Phys. Plasmas 1, 90-95 (1994).

12 -P.A. Gourdain and J.N. Leboeuf, Phys. Plasmas 11, 4372-4381 (2004). 


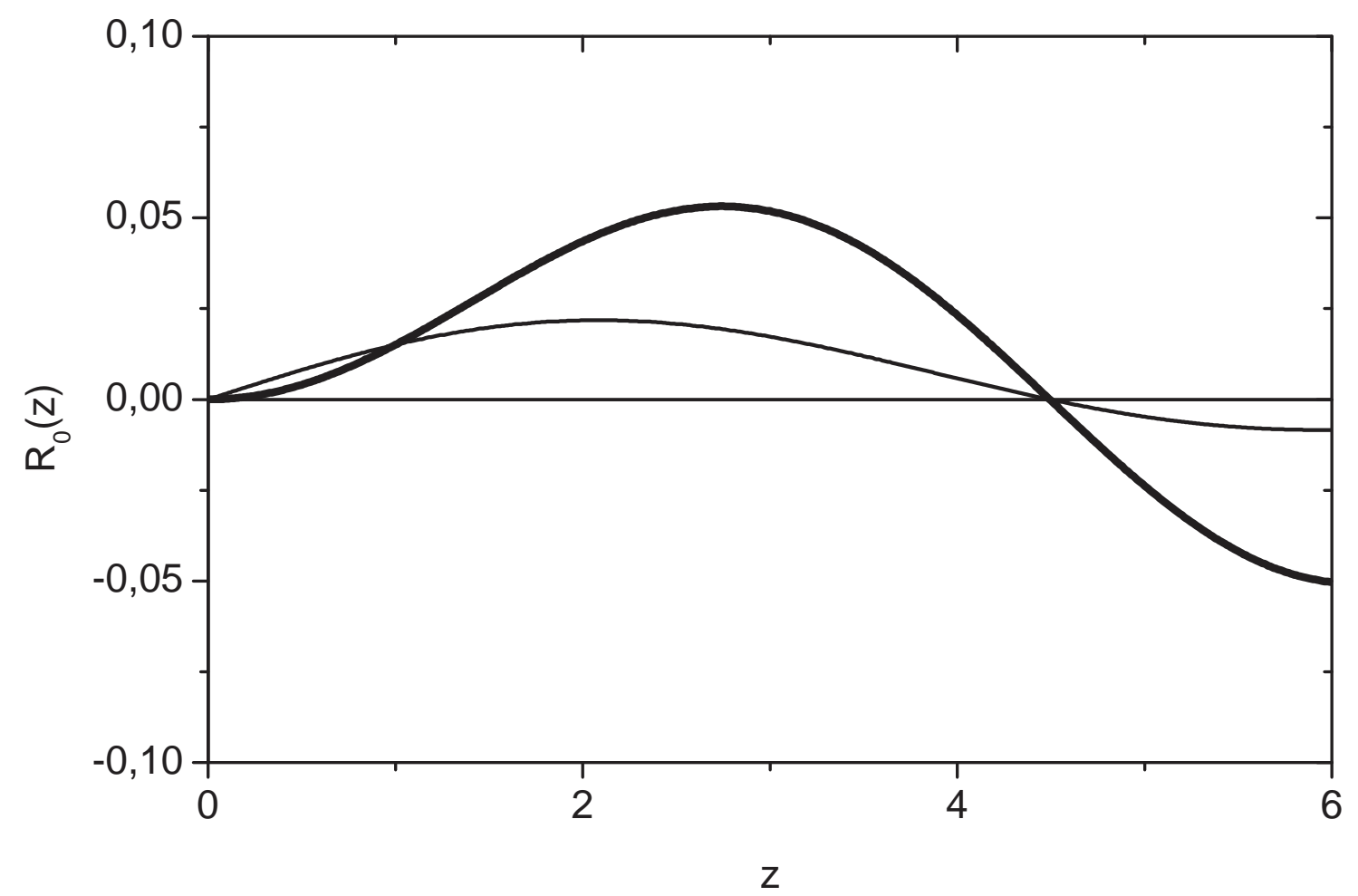

Fig. 1.- The function $R_{0}(z)=A_{0} z j_{1}(z)$ with $A_{0}=0.05$ of the force-free homogeneous radial solution is plotted as a function of $z$ in thick line. The spherical Bessel function $A_{0} j_{1}(z)$ is also plotted in thin line for comparisons. 


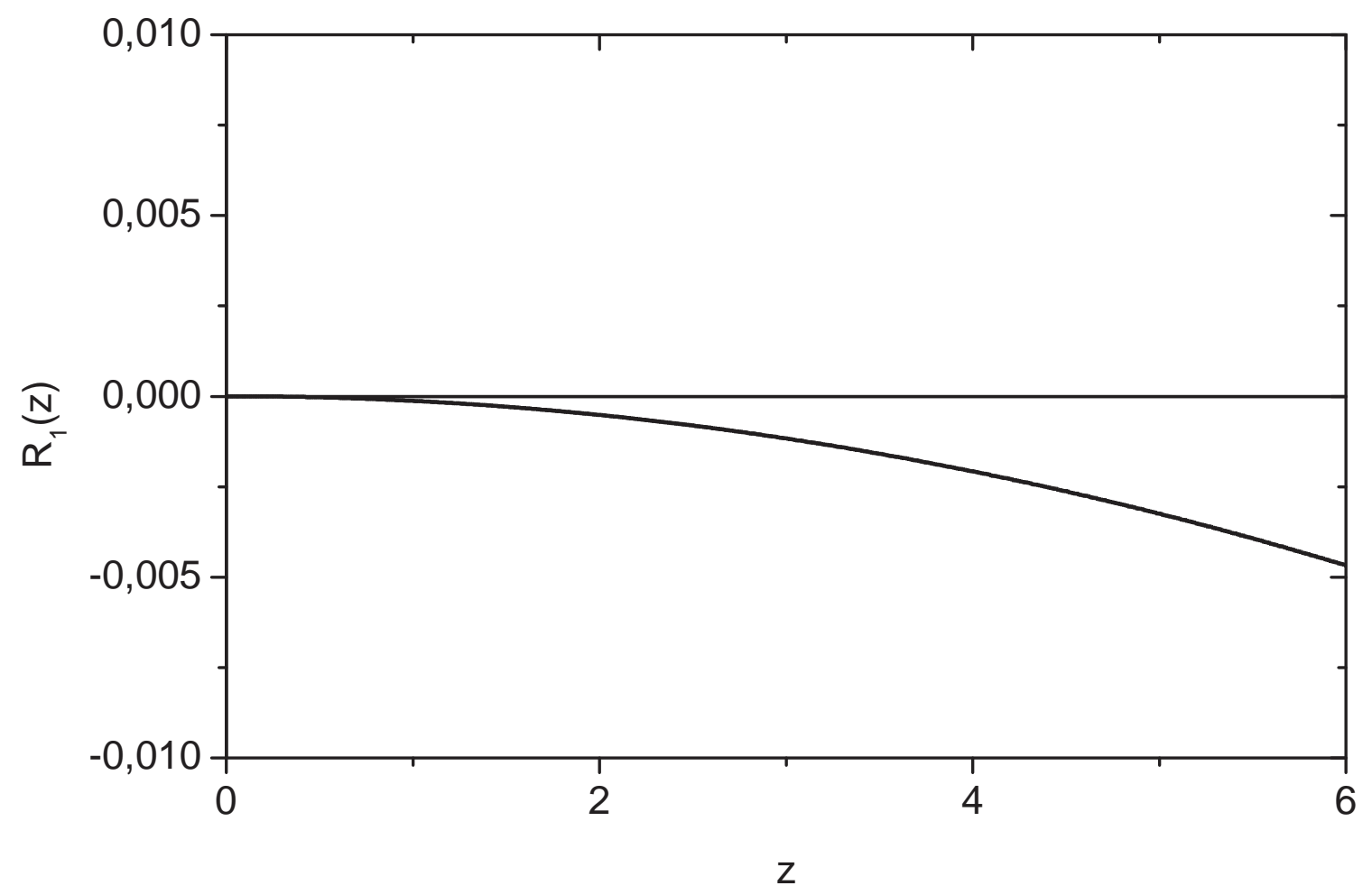

Fig. 2.- The function $R_{1}(z)=-A z^{2}$ with $p_{0}=10^{5} \mathrm{~Pa}$ and $A=1.3 \times 10^{-4}$ of the particular radial solution is plotted as a function of $z$ to show the plasma pressure effect. 


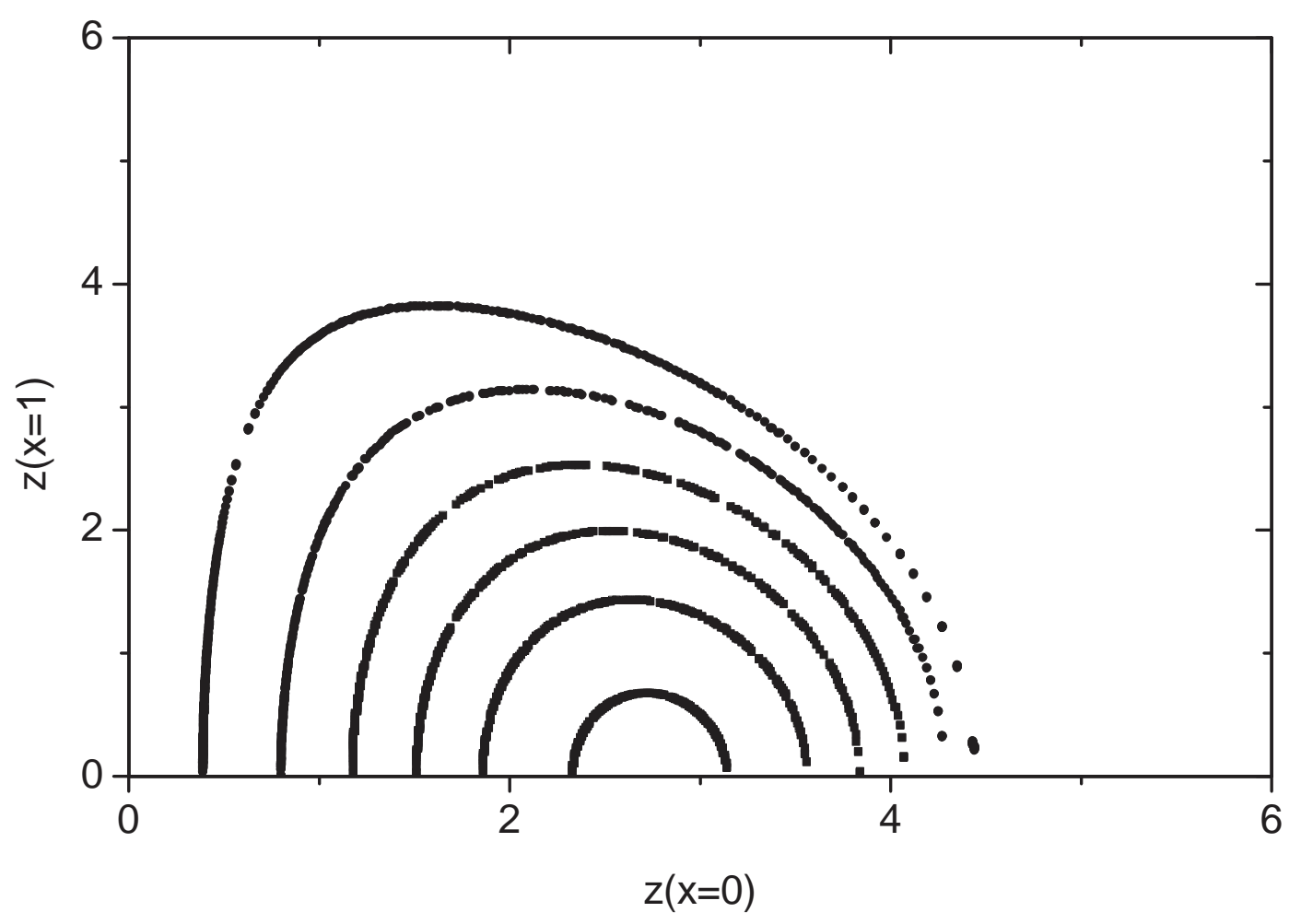

Fig. 3.- The magnetic field lines for spherical tokamak are shown with increasing contour labels from outer to inner contours. These contours are shared by the plasma pressrue and the toroidal field line integral. The axes are labelled in $z$ together with $x=\cos \theta$. 


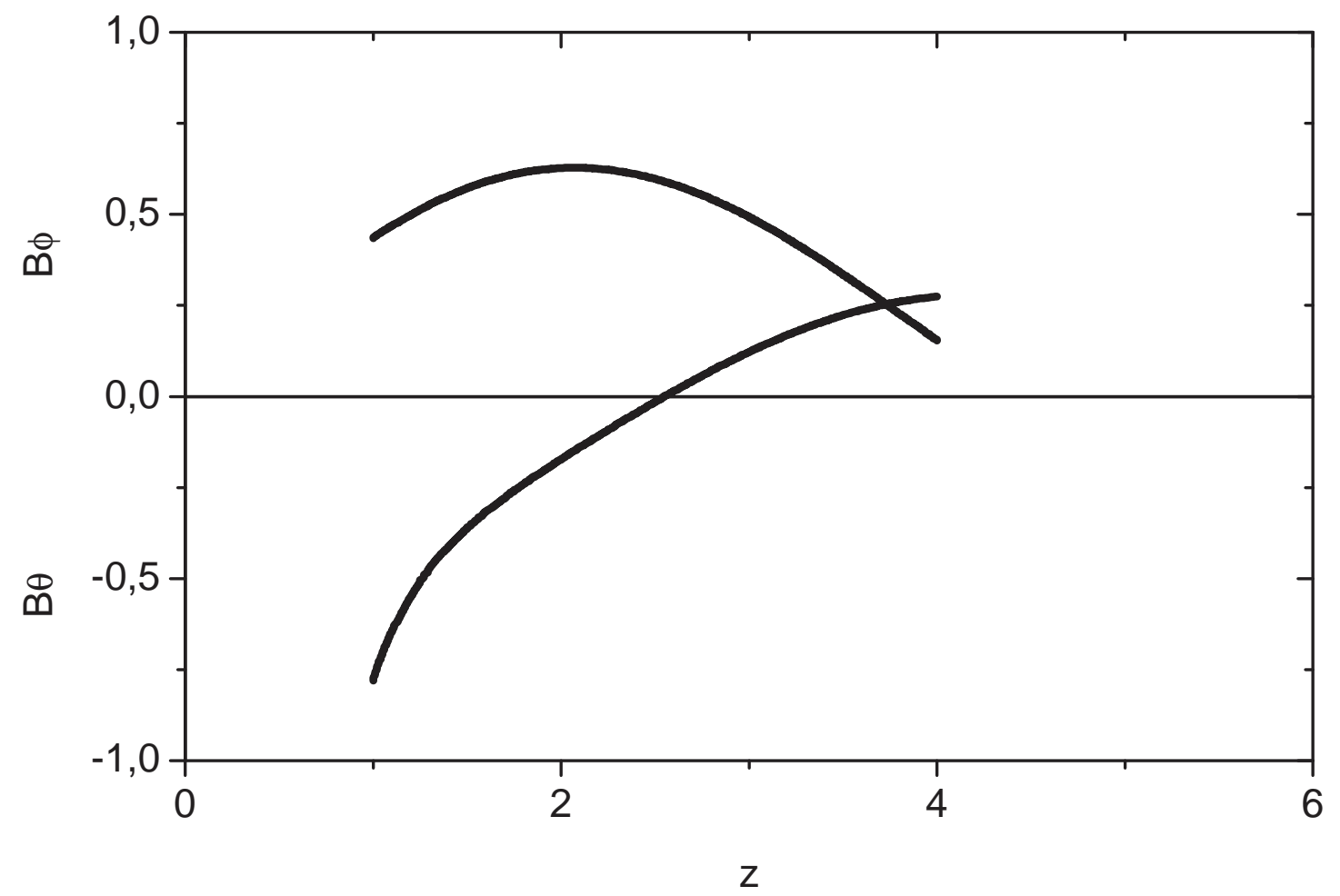

Fig. 4. - The toroidal and the poloidal magnetic fields, $B_{\phi}$ and $B_{\theta}$, with $Q=a P$ and $a=5.4$ are shown as a function of $z$ along $x=0$. 


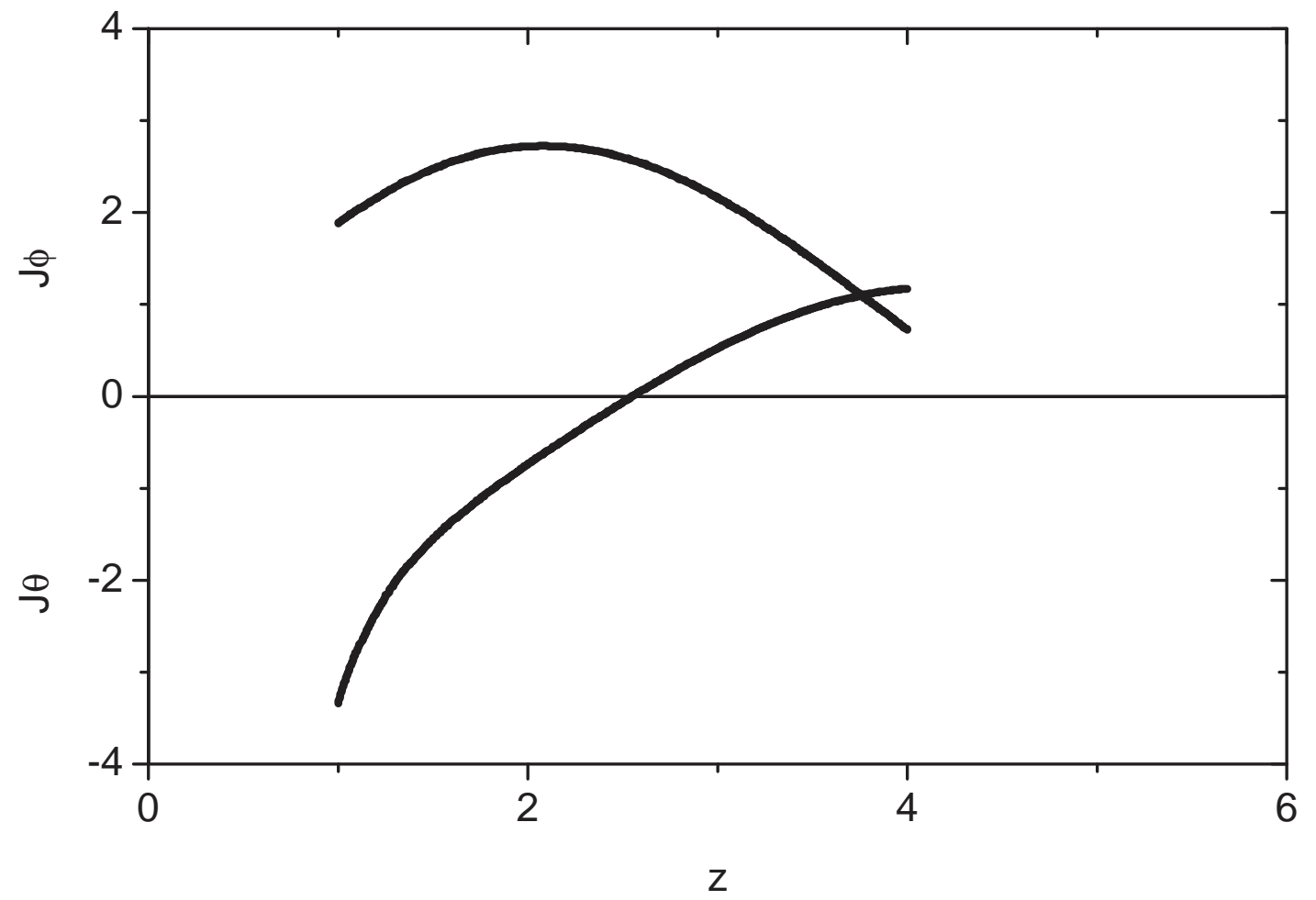

Fig. 5.- The toroidal and the poloidal current densities, $J_{\phi}$ and $J_{\theta}$, with $Q=a P$ and $a=5.4$ are shown as a function of $z$ along $x=0$ in units of $10^{6} \mathrm{~A} / \mathrm{m}^{2}$. 


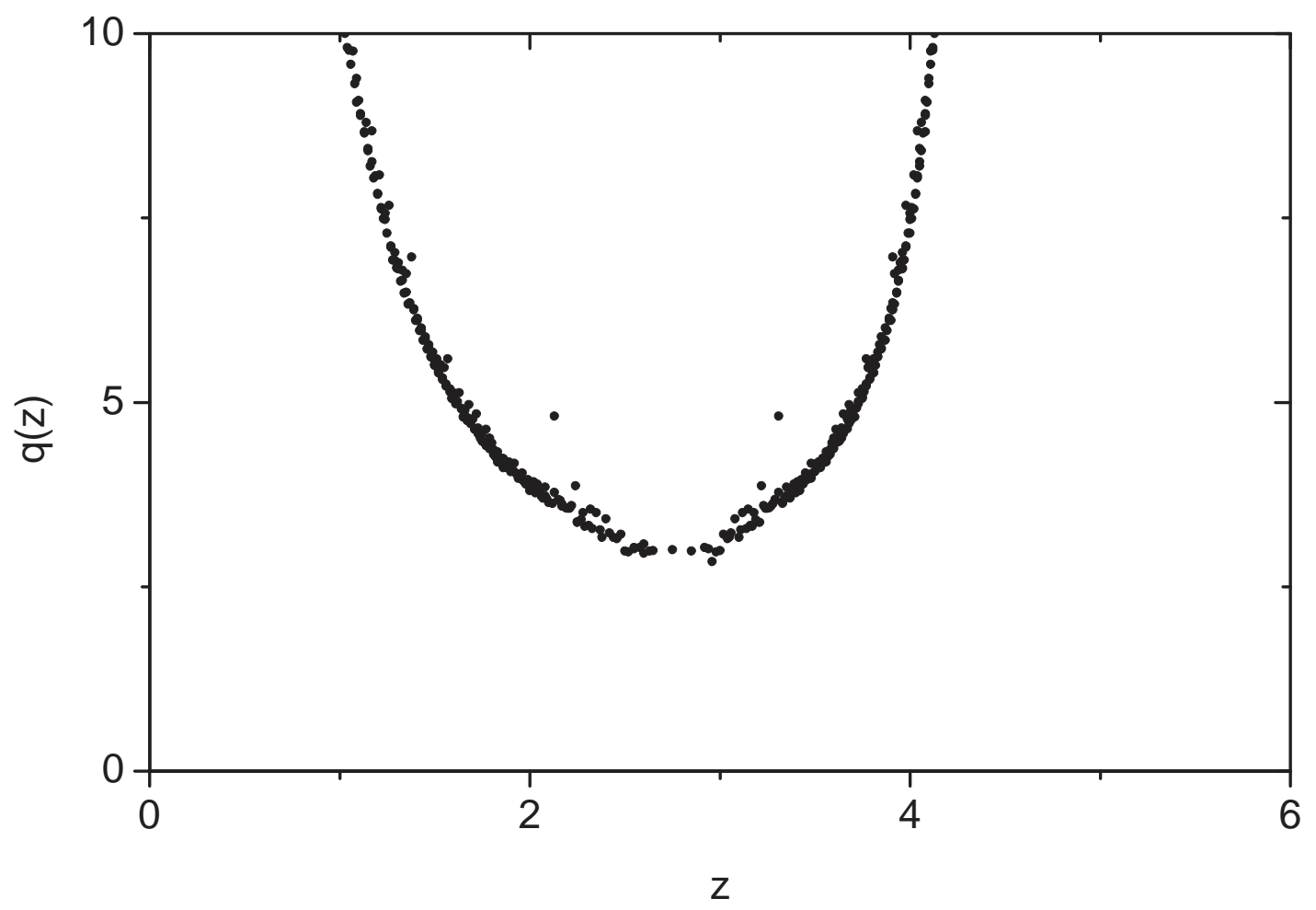

Fig. 6. - The safety factor profile of the plasma equilibrium with nonlinear force-free homogeneous solution, $\gamma^{2}=0.01$, is shown as a function of $z$ along $x=0$. 